\title{
ON THE ORIGIN OF SUPERNOVAE OF TYPE Ib
}

\author{
David Branch \\ Department of Physics and Astronomy, University of Oklahoma \\ Norman, Oklahoma 73019, U.S.A.
}

\section{INTRODUCTION}

The issue I want to discuss is this: supernovae observed during their photospheric phases display three distinct kinds of spectra - but there are four distinct chemical compositions that should be considered for the outer layers of exploding stars. What is the correspondence between the observed supernova type and the chemical composition?

The three kinds of observed spectra are Type II, which by definition shows optical hydrogen lines, Type Ia, which has neither hydrogen nor helium lines, and Type Ib, which has helium but not hydrogen.

The compositions to be considered are hydrogen-rich, or, speaking loosely, "solar"; helium-rich, or, loosely, "Wolf-Rayet"; deflagration - initially a mixture of heavy elements from carbon to radioactive nickel, which decays through cobalt to iron; and detonation - initially a mixture of just helium and radioactive nickel.

By definition, a Type II spectrum is to be associated with a hydrogen-rich composition. Type Ia, lacking hydrogen and helium, is identified with the deflagration composition. Detailed hydrodynamical models of carbon deflagrations in accreting white dwarfs lead to good agreement between theoretical and observed "early-time" photospheric spectra, "late-time" nebular spectra, and light curves. In spite of the successes, the association of Type Ia with deflagrations should be tentative, in view of some unresolved issues such as whether the requisite number of white dwarfs can grow to the Chandrasekhar mass and whether the high predicted gamma-ray flux is really emitted. At present, however, no other model predicts the appropriate heavy-element composition in the outer layers.

The question becomes - which composition, helium-rich or detonation, corresponds to Type $\mathrm{Ib}$ ?

\section{TYPE Ib: MASSIVE-STAR CORE-COLLAPSE OR WHITE-DWARF DETONATION?}

Three fundamental constraints on the nature of Type $\mathrm{Ib}$ are the presence of strong optical lines of He I during the photospheric phase (Harkness et al. 1987); the presence of strong forbidden lines of oxygen ions during the nebular phase (Gaskell et al. 1986, Filippenko and Sargent 
1986); and the association with regions of recent star formation (Porter and Filippenko 1987). These constraints point immediately to the possibility that Type Ib result from the collapsing cores of massive stars that have lost their hydrogen envelopes, i.e., Wolf-Rayet stars (Wheeler and Levreault 1985, Begelman and Sarazin 1986, Chevalier 1986, Filippenko and Sargent 1986, Gaskell et al. 1986, Schaeffer, Casse, and Cahen 1987). The existence of an oxygen layer beneath a helium layer is natural, and massive stars undoubtedly will die in regions of star formation. Uomoto (1986) has discussed the closely related possibility that Type $\mathrm{Ib}$ are core-collapses of helium-rich stars that have lost their hydrogen via Roche-lobe transfer to close companions. Single Wolf-Rayet stars evidently come from stars whose initial mass exceeded $40 \mathrm{M}_{\odot}$ (Abbott et al. 1986) while the loss of hydrogen in a binary can happen to less massive stars.

An alternative way to match the fundamental constraints, involving a thermonuclear explosion in an accreting white dwarf, was suggested by Branch and Nomoto (1986). The motivation was not only to account for Type Ib but also to solve the problem of the "double detonations". The outcome of idealized one-dimensional (spherically-symmetric) models of accreting carbonoxygen white dwarfs depends strongly on the mass accretion rate (Nomoto 1987, Woosley and Weaver 1986). At rates of $10^{-8}-10^{-6} \mathrm{M}_{\odot} \mathrm{yr}^{-1}$ central ignition of degenerate carbon leads to a carbon deflagration and a Type Ia supernova. For rates below $10^{-0} \mathrm{M}_{\odot} \mathrm{yr}^{-1}$ hydrogen shell ignition probably produces a classical nova. At intermediate rates of $10^{-9}-10^{-8} \mathrm{M}_{\odot} \mathrm{yr}^{-1}$, ignition of degenerate helium leads to an outwards detonation wave and, for most parameter choices (Woosley, Taam, and Weaver 1986), an inwards detonation through the CO core; together the two detonations disrupt and eject the entire star as a helium-nickel mixture. With more than a solar mass of radioactive nickel this double-detonation explosion would be bright and easy to see, yet no intrinsically bright supernova with only helium, nickel, and its decay products has been observed. Nature must have a way of suppressing the double-detonations.

Branch and Nomoto speculated that when two-dimensional calculations are carried out, to treat the realistic case of ignition at a point at the base of the helium layer rather than simultaneous ignition all around the spherical base of the layer, the double-detonations might not occur. Instead, the inwards detonation may fail, and the CO core may be left behind as a white dwarf or it may be ejected unburned. The latter case would correspond to a helium-nickel mixture in the outermost layers with a carbon-oxygen mixture beneath - possibly corresponding to Type Ib. Iben et al. (1987) have discussed a similar model in which the accreted mass is helium, rather than hydrogen. Kokhlov and Ergma (1987) have discussed a failed detonation in a low-mass helium white dwarf, but this model wouldn't provide the oxygen layer beneath the helium. 


\section{OBSERVATIONAL CONSTRAINTS}

In this section the Wolf-Rayet and white-dwarf models are discussed in the light of some of the observational constraints. Not all constraints are considered here; for example, the Type Ia infrared light curve shows a distinct dip a few weeks after maximum that is not seen in Type Ib (Elias et al. 1985), but since the cause of the dip in Type Ia is not known its absence in Type Ib does not now provide a useful constraint.

\section{i) The Association with Star-Formation Regions}

Type Ib supernovae appear in or near regions of star formation in spiral galaxies (Porter and Filippenko 1987). However, Huang (1987) has found that Type II supernovae, most of which probably have main sequence masses between 8 and $15 \mathrm{M}_{\odot}$, also occur in star-formation regions. Therefore this constraint is consistent with but does not require the hypothesis that Type Ib have more massive progenitors than Type II. Type Ib also could have somewhat less massive progenitors than Type II. In the white-dwarf model, the initial masses are not expected to be low. If the white dwarf accretes at $10^{-8} \mathrm{M}_{\odot} \mathrm{yr}^{-1}$ at low efficiency (a few percent) from the wind of a companion, and the white dwarf needs to accumulate a few tenths of a solar mass of helium before it explodes, then the companion must be able to lose several solar masses in its wind. It would need to be initially a star of at least intermediate mass $\left(\geq 5 \mathrm{M}_{\odot}\right)$.

\section{ii) The Radio Emission}

The Type Ib supernovae 1983n and 19841 were observed as radio sources (Sramek, Panagia, and Weiler 1984, Panagia, Sramek, and Weiler 1986). Chevalier (1984a) was able to account for the radio emission from SN 1983n using the circumstellar-interaction theory he had introduced to account for Type II supernovae (Chevalier 1984b). Matching the theory to observation gives an estimate of the density in the circumstellar shell, or equivalently an estimate of the ratio of the pre-supernova mass-loss rate to the wind velocity, $\dot{M} / V_{w}$. Chevalier's estimate for SN $1983 \mathrm{n}$ was $5 \times 10^{-7} \mathrm{M}_{\odot} \mathrm{yr}^{-1} / \mathrm{km} \mathrm{s}^{-1}$. In the white-dwarf model the wind of the red-giant donor might be $10 \mathrm{~km} \mathrm{~s}^{-1}$, so the wind mass-loss rate would have to be $5 \times 10^{-6} \mathrm{M}_{\odot} \mathrm{yr}^{-1}$, which is plausible. In the Wolf-Rayet model the wind velocity might be $2000 \mathrm{~km} \mathrm{~s}^{-1}$, so the mass-loss rate would have to be $10^{-3} \mathrm{M}_{\odot} \mathrm{yr}^{-1}$. Observed mass-loss rates for Wolf-Rayet stars are less than $10^{-4} \mathrm{M}_{\odot}$ $\mathrm{yr}^{-1}$ (Abbott et al. 1986). Considering the uncertainties, this discrepancy does not exclude the Wolf-Rayet model, but contrary perhaps to the prevailing wisdom the white-dwarf model has less difficulty with the radio emission than the Wolf-Rayet model, not more. 
iii) The Shape of the Light Curve

The shape of the Type Ib light curve is similar to that of Type Ia. Since the width of the light curve is sensitive principally to the ejected mass, and the carbon-deflagration model gives a good account of the Type Ia light curve with an ejected mass of $1.4 \mathrm{M}_{\odot}$ (Graham 1987), it is not surprising that the predicted light curve for a detonation model that leaves a $\mathrm{CO}$ white dwarf behind is too fast (Woosley, Taam, and Weaver 1986). Whether the predicted light curve will be slowed down when the velocity-sensitive line opacity and the point explosion are correctly treated remains to be seen. Similarly, it is not surprising that the predicted light curve for an $8 \mathrm{M}_{\odot}$ Wolf-Rayet model (Schaeffer, Casse, and Cahen 1987), which ejects $6.6 \mathrm{M}_{\odot}$, is much too broad for Type Ib; at late times the gamma rays from ${ }^{56} \mathrm{Co}$ decay are trapped and the optical light curve follows the ${ }^{56} \mathrm{Co}$ decay (Figure 1 ). If Wolf-Rayet stars produce Type $\mathrm{Ib}$, they must reduce to $4 \mathrm{M}_{\odot}$ or less before they explode!

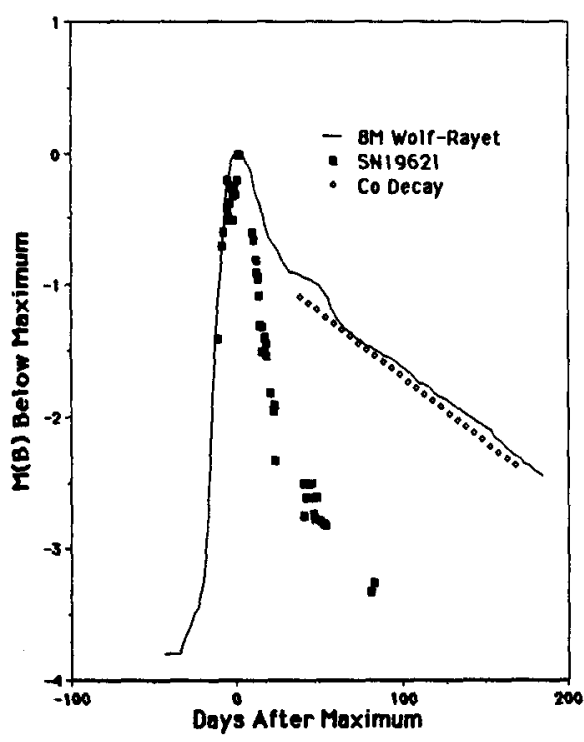

Fig. 1 - The theoretical light light curve for the explosion of an $8 \mathrm{M}_{\odot}$ Wolf-Rayet star (Schaeffer, Casse, and Cahen 1987) is compared to the observed light curve of the Type Ib SN 1962 $\ell$ (Rust 1974), and to the rate of energy released by ${ }^{56} \mathrm{Co}$ decay. Vertical normalization is arbitrary.

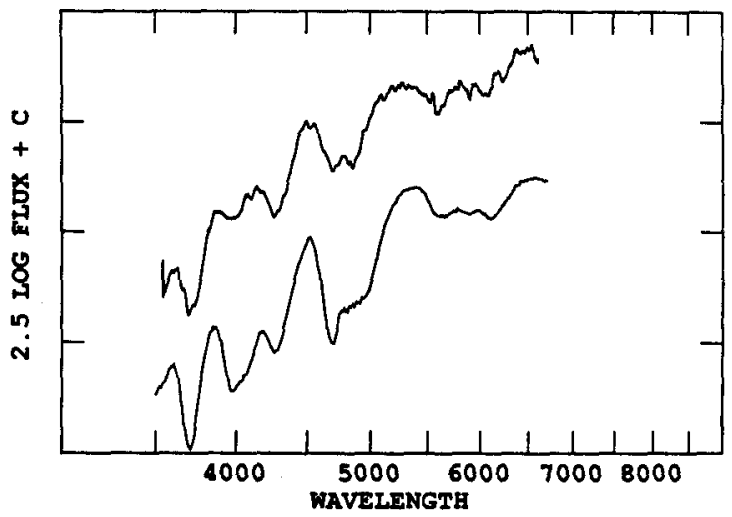

Fig. 2 - The observed pre-maxmimum spectrum of the Type Ib SN $1983 \mathrm{v}$ obtained at the AAT by Axon, Cannon, and McDowell (top) is compared to a synthetic spectrum consisting of lines of Fe II, $\mathrm{He} \mathrm{I}$, and $\mathrm{Ca}$ II and having a velocity at the photosphere of $18,000 \mathrm{~km} \mathrm{~s}^{-1}$ (bottom). 
iv) Spectra During the Photospheric Phase

Harkness et al. (1987) have argued that the optical spectra of Type Ib during the photospheric phases are consistent with a helium-rich composition but not with a detonation composition. I don't think the spectrum is sufficiently well understood to discriminate between the two possibilities. I have calculated the predicted LTE line optical depths at the photosphere for both the helium-rich and detonation compositions, as described in Branch (1987) for the hydrogen-rich composition. Neither composition predicts He I lines to be as strong as observed, so a strong non-LTE excitation of He I needs to be invoked. The reason for a large non-LTE effect in the Wolf-Rayet model is not obvious; a possibility that needs to be checked for the white-dwarf model is non-LTE excitation caused by the decay products of the radioactive cobalt that coexists with the helium.

Strong Fe II lines are predicted for both compositions. One might think that it would be easy to discriminate between the two compositions on the basis of the the lines that accompany the Fe II and He I lines. The problem is that the Fe II lines are ubiquitous - they are all over the optical spectrum and it is not easy to be sure what other lines are present.

Fe II lines also are predicted to dominate the near ultraviolet, for both compositions. A choice between compositions could be made based on the near infrared, where $\mathrm{Mg}$ II and $\mathrm{Ca}$ II lines are stronger than Fe II in the helium-rich case, but no infrared spectra have yet been observed for Type Ib.

An important constraint from the optical photospheric spectra that does not depend on the quantitative interpretion of line strengths is the presence of high-velocity matter just before maximum light. A spectrum of the Type Ib SN 1983 in NGC 1365, obtained at the AAT about 5 days before maximum light by Axon, Cannon, and McDowell, is compared in Figure 2 with a synthetic spectrum based on a velocity at the photosphere of $18,000 \mathrm{~km} \mathrm{~s}^{-1}$. From the fact that the kinetic energy of the matter above the photosphere scales as $t^{2} v^{4}$, it can be shown that this high-velocity phase must have been shortly (a week or less) after the explosion. Otherwise the kinetic energy would be much greater than $10^{51}$ ergs. This adds to the evidence that the ejected mass is low; not only is the light-curve peak narrow, the rise time is short. If the absorption near $3700 \mathrm{~A}$ is due to $\mathrm{Ca}$ II, as it is in the synthetic spectrum, then the detonation would need to falter (Khokhlov and Ergma 1987) in the outermost layers, to leave a small amount of calcium.

v) Spectra During the Nebular Phase

At this meeting Axelrod and Fransson both have shown synthetic nebular spectra based on the Wolf-Rayet model, and both find good qualitative agreement with observation. The main quantitative problem seems to be that since in this model the oxygen is concentrated into a 
shell, the line profiles come out to be box-shaped and broad compared to the narrow centrallypeaked observed lines. Whether this is a fundamental problem with the model or whether it is evidence that mixing of oxygen to deeper slower layers occurs during the explosion is not yet clear. Axelrod and Fransson both have expressed doubt that synthetic nebular spectra for the white-dwarf detonation model would match the observations, although the calculations have not yet been carried out. One virtue of the white-dwarf model is that the oxygen distribution is centrally peaked. One problem is that $\mathrm{CO}$ white dwarfs may contain too much carbon to be consistent with the relatively weak carbon lines in the the observed spectrum (C. Fransson, private communication). Another may be that the inner oxygen region will not absorb enough of the radioactive decay energy to keep the light curve bright and the spectrum dominated by oxygen lines (P. Pinto, private communication).

\section{SUMMARY}

It is not evident which (if either) of the two models discussed here really produces Type Ib. The main problem with the Wolf-Rayet model appears to be that the light-curve width and the high-velocity pre-maximum spectrum demand that the ejected mass be small. Since the consequences for the white-dwarf model of a point ignition have not yet been explored, the white dwarf idea is really a speculation rather than a model. The main problem may be with the nebular spectrum. Although I have argued that the spectrum is not sufficiently well understood to provide a clear choice between the helium-rich and detonation compositions, I think that the situation will improve in the near future. The rapid development of quantitative spectroscopy of supernova photospheric spectra that has been stimulated by the high-quality observations of SN 1987a will lead before long to stringent tests of both models.

\section{ACKNOWLEDGEMENTS}

I have enjoyed lively and informative discussions with many colleagues about the observations and the nature of Type $\mathrm{Ib}$ supernovae. I am especially grateful to Russell Cannon for sending me the spectrum of SN 1983v. This work has been supported by NSF grant AST 8620310.

\section{REFERENCES}

Abbott, D., Beiging, J. H., Churchwell, E., and Torres, A. 1986, Ap. J., 303, 239.

Begelman, M. C., and Sarzin, C. L. 1986, Ap. J. (Lett.), 302 , L59.

Branch, D., and Nomoto, K. 1986, Astr. Ap., 164, L13.

Chevalier, R. A. 1984a, Ap. J. (Lett.), 285, L63.

Chevalier, R. A. 1984b, Ann. N. Y. Acad. Sci., 422, 215.

Chevalier, R. A. 1986, Highlights Astron., 7, 599.

Elias, J. H., Mathews, K., Neugebauer, G., and Persson, S. E. 1986, Ap. J., 296, 379. 
Filippenko, A. V., and Sargent, W. L. W. 1986, A. J., 91, 691.

Gaskell, C. M., Capellaro, E., Dinerstein, H., Garnett, D., Harkness, R. P., and Wheeler, J. C. 1986, Ap. J. (Lett.), 306, L77.

Graham, J. R. 1987, Ap. J., 315, 588.

Harkness, R. P., Wheeler, J. C., Margon, B., Downes, R. A., Kirshner, R. P., Uomoto, A., Barker,

E. S., Cochran, A. L., Dinerstein, H. L., Granett, D. R., and Levreault, R. M. 1987, Ap. J., 317, 355.

Huang, Y.-L. 1987, P.A.S.P., 99, 461.

Iben, I., Jr., Nomoto, K., Tornambe, A., and Tutukov, A. V. 1987, Ap. J., 317, 717.

Khokhlov, A. M., and Ergma, E. V. 1986, Sov. Astr. Lett., 12, 152.

Nomoto, K. 1987, in Proc. 13th Texas Symp. Rel. Ap., ed. M. P. Ulmer (World Scientific Press, Singapore), in press.

Panagia, N., Sramek, R. A., and Weiler, K. W. 1986, Ap. J. (Lett.), 300, L55.

Porter, A. C., and Filippenko, A. V. 1987, A. J., 93,1372.

Rust, B. W. 1974, Oak Ridge Nat. Lab., Rept. 4953.

Schaeffer, R., Casse, M., and Cahen, S. 1987, Ap. J. (Lett.), 316, L31.

Sramek, R. A., Panagia, N., and Weiler, K. W. 1984, Ap. J. (Lett.), 285, L59.

Wheeler, J. C., and Levreault, R. 1985, Ap. J. (Lett.), 294, L17.

Woosley, S. E., Taam, R. E., and Weaver, T. A. 1986, Ap. J., 301, 601.

Woosley, S. E., and Weaver, T. A. 1986, Ann. Rev. Astr. Ap., 24, 205. 